\title{
Robust Control of Switched Linear Systems with Output Switching Strategy
}

\author{
Edson Italo Mainardi Júnior ${ }^{1}$ - Marcelo Carvalho Minhoto Teixeira ${ }^{3}$. \\ Rodrigo Cardim ${ }^{3}$ - Edvaldo Assunção ${ }^{3}$ - Manoel Rodrigo Moreira ${ }^{3}$. \\ Diogo Ramalho de Oliveira $^{3}$. Alexandre Ataide Carniato ${ }^{2}$
}

Received: 19 December 2014 / Revised: 29 May 2015 / Accepted: 19 June 2015 / Published online: 7 July 2015

(C) Brazilian Society for Automatics-SBA 2015

\begin{abstract}
This paper investigates the robust control problem of continuous-time uncertain switched linear systems, using only a switching strategy depending on the plant output. The proposed method is based on linear matrix inequalities (LMIs). A set of slack variables is introduced to reduce the design conservatism, and new sufficient LMI conditions for the synthesis of the controllers are presented. Two examples show that the proposed method has an adequate performance even in situations when the matrices of the linear subsystems
\end{abstract}

Edson Italo Mainardi Júnior

edsonitalo@yahoo.com.br

Marcelo Carvalho Minhoto Teixeira

marcelo@dee.feis.unesp.br

Rodrigo Cardim

rcardim@dee.feis.unesp.br

Edvaldo Assunção

edvaldo@dee.feis.unesp.br

Manoel Rodrigo Moreira

manoel.rodrigo@hotmail.com

Diogo Ramalho de Oliveira

diogo_oliveira6@hotmail.com

Alexandre Ataide Carniato

carniato@ifsp.edu.br

1 Instituto Federal de Educação, Ciência e Tecnologia Catarinense (IFC), Campus Videira, Rodovia SC 135, Videira, SC 89560-000, Brazil

2 Instituto Federal de Educação, Ciência e Tecnologia de São Paulo (IFSP), Campus Presidente Epitácio, Av José Ramos Júnior, 27-50, Presidente Epitácio, SP 19470-000, Brazil

3 Laboratório de Pesquisa em Controle (LPC), Departamento de Engenharia Elétrica, Faculdade de Engenharia, Univ Estadual Paulista (UNESP), Campus Ilha Solteira, Avenida José Carlos Rossi, 1370, Ilha Solteira, SP 15385-000, Brazil are not Hurwitz and offers a simple and efficient solution for this control problem.

Keywords Switched linear systems - Quadratic Lyapunov function - Output switching strategy - Linear matrix inequalities $\cdot$ Robust control with polytopic uncertainties

\section{Introduction}

In recent decades, the scientific community has shown a growing interest in the study of the stability of switched linear systems (Geromel and Colaneri 2006; Zhai et al. 2003). This interest is mainly due to the fact that they usually allow a better overall performance, namely their applications in practical systems such as: mechanical control systems, process control, power systems, aircraft control, automotive industry, power electronics (Yoshimura et al. 2013; Scharlau et al. 2014; Mainardi Júnior et al. 2012a; Cardim et al. 2009; Deaecto et al. 2010). In general, the theory of switched linear systems can be divided into two groups, where the first one occurs when the switching strategy $\sigma(t)$ is independent of the system state variables and the second one occurs when $\sigma(t)$ is a control variable dependent on the state variables of the system (Geromel and Colaneri 2006). The main goal of this paper is to design an appropriate output switching strategy that selects at each instant of time a dynamic subsystem among a determined number of available subsystems, which provides asymptotic stability to a given equilibrium point of the controlled system, with the assurance of an adequate performance (Decarlo et al. 2000; Sun and Ge 2005b). Important results are presented in Lin and Antsaklis (2009) and Liberzon (2003). See Hespanha and Morse (2002) for the synthesis of controllers that stabilize switched linear systems in a general context. In Wicks et al. (1994), the stability problem of 
switched linear systems was studied using a quadratic Lyapunov function, and it has been shown that if there exists a Hurwitz convex combination of the subsystem matrices (where a matrix with all eigenvalues with negative real parts is obtained), then there exists a state-dependent switching strategy that stabilizes the switched linear system. Within this context, in Feron (1996), the author demonstrated that the condition shown in Wicks et al. (1994) is necessary and sufficient for two subsystems. Furthermore, in Feron (1996) an extension of these results, using dynamic output feedback and based on a quadratic Lyapunov function, was also presented.

For robust stabilization, in Zhai et al. (2003) a quadratic stabilizing switching law was designed for polytopic uncertain switched linear systems, based on LMIs. Next, in Ji et al. (2003) two sufficient conditions were proposed, and more specifically, the quadratic stabilization of switched linear systems consider a switching strategy added to the state feedback controllers or to the output feedback controllers. Other results that investigate problems about stability and stabilizability of continuous-time and discrete-time switched systems with uncertainties can be found in Lin and Antsaklis (2007) and Otsuka and Soga (2010). A brief review and results on controllability, observability, controller design and optimal control of switched linear systems can be found in Sun and Ge (2005a).

In Geromel et al. (2008), stability conditions were analyzed and a switching strategy was proposed together with the controller design using dynamic output feedback for a particular class of matrix inequalities called Lyapunov-Metzler inequalities, for both continuous-time and discrete-time plants. Accordingly, Deaecto et al. (2011) presented recent results considering the $H_{\infty}$ norm for designing controllers via dynamic output state feedback.

The main reason for this paper is the control design of uncertain switched linear systems, considering only output access for the switching strategy design. This is due to the fact that the design of static control, considering only the output of the plant, allows a simple practical implementation. In Iwasaki et al. (1994), an alternative method was presented for a continuous-time linear system, for designing a stabilizing static output feedback controller which ensures a specified linear quadratic (LQ) performance. Next, necessary but not sufficient conditions were presented in Geromel et al. (1998). Important results for this system class can be also found in Syrmos et al. (1994).

Additionally, in the control problem via static output feedback, two different LMI-based conditions were proposed in Daafouz et al. (2001) and Daafouz et al. (2002), based on a switched quadratic Lyapunov function, where the first one is classical and the second one is new and uses a slack variable, which makes it useful for designing problems. More recently, Ding and Yang (2009) describes more relaxed con- ditions using the Finsler's lemma and piecewise quadratic Lyapunov functions. Other important results of controller designs via static output feedback can be found in Dong and Yang (2007).

A new static control strategy, for switched linear system without uncertainties, was presented in Mainardi Júnior et al. (2012b), considering available only the plant output. In Mainardi Júnior et al. (2014), the output static control strategy described in Mainardi Júnior et al. (2012b) was extended to a class of uncertain switched linear systems. The main goal of this paper is to propose another extension of the output static control strategy presented in Mainardi Júnior et al. (2014), for a class of uncertain switched linear systems. This procedure allows a practical implementation, significantly simpler than those available in the literature, because the plant output is used only for implementing the static switching strategy. In order to reduce conservatism of the design conditions, new sufficient LMIs are proposed in the design.

The stability analysis has been reduced to problems described by LMIs (Boyd et al. 1994) that, when feasible, are easily solved by some tools available in the literature of convex programming (Gahinet et al. 1995). The LMI formulation has been used in several engineering problems such as in the design of regulators for nonlinear systems represented by Takagi-Sugeno fuzzy models (Guedes et al. 2013; da Cruz Figueredo et al. 2013). The notation used is described as follows. For real matrices or vectors, $\left(^{\prime}\right)$ indicates transpose. The set composed by the first $N$ positive integers $\{1, \ldots, N\}$ is denoted by $\mathbb{K}_{N}$. The set of all vectors $\lambda=\left[\lambda_{1} \ldots \lambda_{N}\right]^{\prime}$ such that $\lambda_{i} \geq 0, i \in \mathbb{K}_{N}$ and $\lambda_{1}+\lambda_{2}+\ldots+\lambda_{N}=1$ is denoted by $\Lambda$. The convex combination of a set of matrices $\left(A_{1}, \ldots, A_{N}\right)$ is denoted by $A_{\lambda}=\sum_{i=1}^{N} \lambda_{i} A_{i}$, where $\lambda \in \Lambda$.

\section{Switched Linear Systems}

Consider the switched linear system defined by Geromel and Colaneri (2006):

$\left\{\begin{array}{l}\dot{x}(t)=A_{\sigma} x(t), \quad x(0)=x_{0} \\ y(t)=C x(t)\end{array}\right.$

where $x(t) \in \mathbb{R}^{n}$ is the state vector, $y(t) \in \mathbb{R}^{p}$ is the output, $\sigma(t)$ is the switching strategy, $x_{0}$ is the initial condition, and $C \in \mathbb{R}^{p \times n}$ is the output matrix of the system, constant for all $t \geq 0$. Given a known set of constant matrices $A_{i} \in \mathbb{R}^{n \times n}$, for $i \in \mathbb{K}_{N}$, then the switching strategy $\sigma(t)$ is such that

$A_{\sigma(t)} \in\left\{A_{1}, A_{2}, \ldots, A_{N}\right\}$,

where $A_{\sigma(t)}$ must jump from $A_{i}$ to $A_{j}, i \neq j$, when the switching occurs from $\sigma(t)=i$ to $\sigma(t)=j$. The control problem is the following: design a switching law, such that 
the equilibrium point $x=0$ of the switched linear system (1) is globally asymptotically stable.

A sufficient condition for this control problem is the quadratic stability defined below.

Definition 1 (Otsuka and Soga 2010) The switched linear system (1) and (2) is said to be quadratically stabilizable, if only if there exist a Lyapunov function of the form $V(x)=$ $x^{\prime} P x$, a positive number $\epsilon \in \mathbb{R}^{+}$, and a switching strategy $\sigma(x, t)$ such that

$\dot{V}(x)<-\epsilon x^{\prime} x$,

for all trajectory $x(t)$, of the switched linear system (1) and (2).

Next, an important and known theorem is described, which presents a necessary and sufficient condition for quadratic stability of switched linear systems.

Theorem 1 (Feron 1996) Assume $N=2$. The switched linear system (1) and (2) is quadratically stabilizable if and only if there exists $\lambda \in \Lambda$ such that $\lambda_{1} A_{1}+\lambda_{2} A_{2}$ is Hurwitz.

Proof See Feron (1996) for details.

In order to generalize Theorem 1, the following important lemma was presented in Deaecto et al. (2010).

Lemma 1 (Deaecto et al. 2010) Consider the switched linear system (1) and (2) and suppose that the state vector $x(t) \in \mathbb{R}^{n}$ is available. If there exists $\lambda \in \Lambda$ and a symmetric positive definite matrix $P \in \mathbb{R}^{n \times n}$, such that

$A_{\lambda}^{\prime} P+P A_{\lambda}<0$,

then the switching strategy

$\sigma(x)=\arg \min _{i \in \mathbb{K}_{N}}\left(x^{\prime} P A_{i} x\right)$

makes the origin $x=0$ of the switched linear system (1) and (2) a globally asymptotically stable equilibrium point.

Proof The proof of this result uses a quadratic Lyapunov function $V(x)=x^{\prime} P x$. See Deaecto et al. (2010) for details.

In the next section, the main goal of this study is presented and sufficient conditions for the control of uncertain switched linear systems via an output switching strategy are proposed.

\section{Robust Output Control of Polytopic Uncertain Switched Linear Systems}

Consider the continuous-time uncertain switched linear system defined by the following state-space realization:

$$
\left\{\begin{array}{l}
\dot{x}(t)=A(\alpha, \sigma) x(t), \quad x(0)=x_{0} \\
y(t)=C x(t)
\end{array}\right.
$$

where $x(t) \in \mathbb{R}^{n}$ is the state vector, $y(t) \in \mathbb{R}^{p}$ is the output with $C \in \mathbb{R}^{p \times n}$ a constant matrix, and $x_{0}$ is the initial condition. In the matrix $A(\alpha, \sigma)$, the vector $\alpha=\left[\alpha_{1} \alpha_{2} \ldots \alpha_{r}\right]^{\prime}$ represents the polytopic uncertainties (or structural failures) of the plant and $\sigma(t)$ is the switching strategy. The matrix $A(\alpha, \sigma) \in \mathbb{R}^{n \times n}$ can be described by convex combinations of their vertices, as below:

$$
\begin{gathered}
A(\alpha, \sigma)=\sum_{j=1}^{r} \alpha_{j} A_{\sigma j}, \quad \sum_{j=1}^{r} \alpha_{j}=1, \\
\alpha_{j} \geq 0, \quad \sigma(t) \in \mathbb{K}_{N},
\end{gathered}
$$

where $r$ is the number of vertices of the polytopic uncertainties. For more details about polytopic uncertainties, see Boyd et al. (1994) and Bernussou et al. (1989). Now, the control problem is the following: Considering that $x(t) \in \mathbb{R}^{n}$ is not available but $y(t) \in \mathbb{R}^{p}$ is always available, determine a switching strategy $\sigma(t)$, for all $t \geq 0$, which makes the origin $x=0$ of the polytopic uncertain switched linear system (6) and (7) a globally asymptotically stable equilibrium point. It is not considered that each matrix of the linear subsystems is Hurwitz. A solution for this problem is presented in the following theorem.

Theorem 2 Mainardi Júnior et al. (2014) Consider the uncertain switched linear system (6) and (7), and suppose that the output $y(t) \in \mathbb{R}^{p}$ is always available. If there exist $\lambda \in \Lambda$, symmetric matrices $Q_{0} \in \mathbb{R}^{n \times n}, Q_{i} \in \mathbb{R}^{p \times p}$ and $a$ symmetric positive definite matrix $P \in \mathbb{R}^{n \times n}$, such that

$$
\begin{aligned}
& A_{i j}^{\prime} P+P A_{i j}<Q_{0}+C^{\prime} Q_{i} C, \\
& Q_{0}+C^{\prime} Q_{\lambda} C<0,
\end{aligned}
$$

for all $i \in \mathbb{K}_{N}$ and $j \in \mathbb{K}_{r}$, then the switching strategy

$\sigma(y)=\arg \min _{i \in \mathbb{K}_{N}}\left(y^{\prime} Q_{i} y\right)$

makes the origin $x=0$ of the uncertain switched linear system (6) and (7) a globally asymptotically stable equilibrium point.

Proof See Mainardi Júnior et al. (2014).

In order to relax the feasibility of the LMIs from Theorem 2, a less conservative condition is proposed in Theorem 3, considering that the vector $\alpha=\left[\begin{array}{lll}\alpha_{1} & \alpha_{2} \ldots \alpha_{r}\end{array}\right]^{\prime}$ defined in (7) is uncertain and time-invariant.

Theorem 3 Consider the uncertain switched linear system (6) and (7), and suppose that the output $y(t) \in \mathbb{R}^{p}$ is always available. If there exist $\lambda \in \Lambda$, symmetric matrices $Q_{0 j} \in$ $\mathbb{R}^{n \times n}, Q_{i} \in \mathbb{R}^{p \times p}$ and symmetric positive definite matrices $P_{k} \in \mathbb{R}^{n \times n}$, such that 


$$
\begin{aligned}
& \frac{1}{2} \times\left(A_{i j}^{\prime} P_{k}+P_{k} A_{i j}+A_{i k}^{\prime} P_{j}+P_{j} A_{i k}\right) \\
& \quad<Q_{0 j}+C^{\prime} Q_{i} C, \quad k \leq j, \\
& Q_{0 j}+C^{\prime} Q_{\lambda} C<0,
\end{aligned}
$$

for all $i \in \mathbb{K}_{N}, j \in \mathbb{K}_{r}$ and $k \in \mathbb{K}_{r}$, then the switching strategy (10) makes the origin $x=0$ of the uncertain switched system (6) and (7) a globally asymptotically stable equilibrium point.

Proof Considering the quadratic Lyapunov candidate function, $V(x(t))=\sum_{k=1}^{r} \alpha_{k} x^{\prime} P_{k} x$, where $k=1,2, \ldots, r$. From (10), (6) and (12), one has for $x \neq 0$ :

$$
\begin{aligned}
\dot{V}(x)= & \sum_{k=1}^{r} \alpha_{k} x^{\prime}\left(P_{k} A(\alpha, \sigma)+A^{\prime}(\alpha, \sigma) P_{k}\right) x \\
= & \sum_{k=1}^{r} \alpha_{k} \sum_{j=1}^{r} \alpha_{j} x^{\prime}\left(P_{k} A_{\sigma j}+A_{\sigma j}^{\prime} P_{k}\right) x \\
= & \frac{1}{2} \sum_{k=1}^{r} \alpha_{k} \sum_{j=1}^{r} \alpha_{j} x^{\prime}\left(P_{k} A_{\sigma j}+A_{\sigma j}^{\prime} P_{k}\right. \\
& \left.+P_{j} A_{\sigma k}+A_{\sigma k}^{\prime} P_{j}\right) x \\
< & \sum_{k=1}^{r} \alpha_{k} \sum_{j=1}^{r} \alpha_{j} x^{\prime}\left(Q_{0 j}+C^{\prime} Q_{\sigma} C\right) x \\
= & \sum_{j=1}^{r} \alpha_{j} x^{\prime}\left(Q_{0 j}+C^{\prime} Q_{\sigma} C\right) x \\
= & \sum_{j=1}^{r} \alpha_{j} x^{\prime} Q_{0 j} x+\sum_{j=1}^{r} \alpha_{j} x^{\prime}\left(C^{\prime} Q_{\sigma} C\right) x \\
= & \sum_{j=1}^{r} \alpha_{j} x^{\prime} Q_{0 j} x+x^{\prime}\left(C^{\prime} Q_{\sigma} C\right) x \\
= & \sum_{j=1}^{r} \alpha_{j} x^{\prime} Q_{0 j} x+\min _{i \in \mathbb{K}_{N}}\left(y^{\prime} Q_{i} y\right) \\
\leq & x^{\prime}\left(\sum_{j=1}^{r} \alpha_{j} Q_{0 j}+C^{\prime} Q_{\lambda} C\right) x<0 . \\
&
\end{aligned}
$$

The proof is concluded.

Remark 1 Note that Theorem 2 is a particular case of Theorem 3, because considering $A_{i k}=A_{i j}$ and $P_{k}=P_{j}=P$ for all $j \in \mathbb{K}_{r}$ and $k \in \mathbb{K}_{r}$, then the conditions given by Theorems 2 and 3 are equivalents. Thus, if the conditions of Theorem 2 are feasible, then the conditions of Theorem 3 are also feasible.

In Theorems 4 and 5, less conservative conditions are proposed, toward a generalization of the previous results presented in Mainardi Júnior et al. (2014).
Theorem 4 If there exist $\lambda \in \Lambda$, matrices $X_{1 i} \in \mathbb{R}^{n \times n}$, $X_{2 i} \in \mathbb{R}^{n \times n}$, symmetric matrices $Q_{0 j} \in \mathbb{R}^{n \times n}, Q_{i} \in \mathbb{R}^{p \times p}$ and symmetric positive definite matrices $P_{j} \in \mathbb{R}^{n \times n}$, such that

$$
\begin{aligned}
& {\left[\begin{array}{cc}
X_{1 i} A_{i j}+A_{i j}^{\prime} X_{1 i}^{\prime} & P_{j}-X_{1 i}+A_{i j}^{\prime} X_{2 i}^{\prime} \\
P_{j}-X_{1 i}^{\prime}+X_{2 i} A_{i j} & -X_{2 i}-X_{2 i}^{\prime}
\end{array}\right]} \\
& <\left[\begin{array}{cc}
Q_{0 j}+C^{\prime} Q_{i} C & 0 \\
0 & 0
\end{array}\right], \\
& Q_{0 j}+C^{\prime} Q_{\lambda} C<0,
\end{aligned}
$$

for all $i \in \mathbb{K}_{N}$ and $j \in \mathbb{K}_{r}$, then the switching strategy (10) makes the origin $x=0$ of the uncertain switched linear system (6) and (7) a globally asymptotically stable equilibrium point.

Proof Consider that (14) and (15) are feasible. Thus, from (10) and (15), for $x \neq 0$ it follows that:

$$
\begin{aligned}
0 & >x^{\prime}\left(Q_{0 j}+C^{\prime} Q_{\lambda} C\right) x \geq x^{\prime} Q_{0 j} x+\min _{i \in \mathbb{K}_{N}}\left(y^{\prime} Q_{i} y\right) \\
& =x^{\prime}\left(Q_{0 j}+C^{\prime} Q_{\sigma} C\right) x .
\end{aligned}
$$

Observe that (16) can be rewritten as:

$$
\begin{aligned}
& x\left(Q_{0 j}+C^{\prime} Q_{\sigma} C\right) x=x^{\prime}\left[\begin{array}{ll}
I_{n} & A^{\prime}(\sigma, \alpha)
\end{array}\right] \\
& {\left[\begin{array}{cc}
Q_{0 j}+C^{\prime} Q_{\sigma} C & 0 \\
0 & 0
\end{array}\right]\left[\begin{array}{c}
I_{n} \\
A(\sigma, \alpha)
\end{array}\right] x<0 .}
\end{aligned}
$$

Then, from (14) and (17) note that:

$$
\begin{aligned}
& 0>x^{\prime}\left[\begin{array}{ll}
I_{n} & A^{\prime}(\sigma, \alpha)
\end{array}\right]\left[\begin{array}{cc}
Q_{0 j}+C^{\prime} Q_{\sigma} C & 0 \\
0 & 0
\end{array}\right]\left[\begin{array}{c}
I_{n} \\
A(\sigma, \alpha)
\end{array}\right] x \\
& >x^{\prime}\left[\begin{array}{ll}
I_{n} & A^{\prime}(\sigma, \alpha)
\end{array}\right] \\
& {\left[\begin{array}{cc}
X_{1 \sigma} A_{\sigma j}+A_{\sigma j}^{\prime} X_{1 \sigma}^{\prime} & P_{j}-X_{1 \sigma}+A_{\sigma j}^{\prime} X_{2 \sigma}^{\prime} \\
P_{j}-X_{1 \sigma}^{\prime}+X_{2 \sigma} A_{\sigma j} & -X_{2 \sigma}-X_{2 \sigma}^{\prime}
\end{array}\right]} \\
& {\left[\begin{array}{c}
I_{n} \\
A(\sigma, \alpha)
\end{array}\right] x \text {. }}
\end{aligned}
$$

Now, define $P(\alpha)=\left(\alpha_{1} P_{1}+\alpha_{2} P_{2}+\ldots+\alpha_{r} P_{r}\right)$. Then, from (7), multiplying (18) by $\alpha_{j}$ and taking the sum from $j=1$ to $j=r$, one has:

$$
\begin{aligned}
& 0>x^{\prime}\left[\begin{array}{ll}
I_{n} & A^{\prime}(\sigma, \alpha)
\end{array}\right] \\
& {\left[\begin{array}{cc}
\sum_{j=1}^{r} \alpha_{j}\left(Q_{0 j}+C^{\prime} Q_{\sigma} C\right) & 0 \\
0 & 0
\end{array}\right]\left[\begin{array}{c}
I_{n} \\
A(\sigma, \alpha)
\end{array}\right] x} \\
& >x^{\prime}\left[\begin{array}{ll}
I_{n} & A^{\prime}(\sigma, \alpha)
\end{array}\right]\left[\begin{array}{l}
X_{1 \sigma} A(\sigma, \alpha)+A^{\prime}(\sigma, \alpha) X_{1 \sigma}^{\prime} \\
P(\alpha)-X_{1 \sigma}^{\prime}+X_{2 \sigma} A(\sigma, \alpha)
\end{array}\right. \\
& \left.\begin{array}{c}
P(\alpha)-X_{1 \sigma}+A^{\prime}(\sigma, \alpha) X_{2 \sigma}^{\prime} \\
-X_{2 \sigma}-X_{2 \sigma}^{\prime}
\end{array}\right]\left[\begin{array}{c}
I_{n} \\
A(\sigma, \alpha)
\end{array}\right] x
\end{aligned}
$$




$$
\begin{aligned}
& =x^{\prime}\left[\begin{array}{ll}
I_{n} & A^{\prime}(\sigma, \alpha)
\end{array}\right]\left\{\left[\begin{array}{cc}
0 & P(\alpha) \\
P(\alpha) & 0
\end{array}\right]\right. \\
& +\left[\begin{array}{l}
X_{1 \sigma} \\
X_{2 \sigma}
\end{array}\right]\left[A(\sigma, \alpha)-I_{n}\right] \\
& \left.+\left[\begin{array}{c}
A^{\prime}(\sigma, \alpha) \\
-I_{n}
\end{array}\right]\left[\begin{array}{ll}
X_{1 \sigma}^{\prime} & X_{2 \sigma}^{\prime}
\end{array}\right]\right\}\left[\begin{array}{c}
I_{n} \\
A(\sigma, \alpha)
\end{array}\right] x \\
& =x^{\prime}\left[\begin{array}{ll}
I_{n} & A^{\prime}(\sigma, \alpha)
\end{array}\right]\left[\begin{array}{cc}
0 & P(\alpha) \\
P(\alpha) & 0
\end{array}\right]\left[\begin{array}{c}
I_{n} \\
A(\sigma, \alpha)
\end{array}\right] x \text {. }
\end{aligned}
$$

Considering a Lyapunov function candidate $V(x)=$ $x^{\prime} P(\alpha) x$, note that from (7), $V(x)>0$ for $x \neq 0$ and from (6) and (19) it follows that $\dot{V}(x)<0$ for $x \neq 0$. The proof is concluded.

Now, in order to relax the feasibility of the LMIs from Theorem 4, a less conservative condition is proposed in Theorem 5, considering also that the vector $\alpha=\left[\begin{array}{lll}\alpha_{1} & \alpha_{2} \ldots \alpha_{r}\end{array}\right]^{\prime}$ defined in (7) is uncertain and time-invariant. These new sufficient LMI conditions reduce the conservatism of the results shown in Theorem 4.

Theorem 5 If there exist $\lambda \in \Lambda$, matrices $X_{1 i k} \in \mathbb{R}^{n \times n}$, $X_{2 i k} \in \mathbb{R}^{n \times n}$, symmetric matrices $Q_{0 j k} \in \mathbb{R}^{n \times n}, Q_{i} \in$ $\mathbb{R}^{p \times p}$ and symmetric positive definite matrices $P_{j k} \in \mathbb{R}^{n \times n}$, such that

$$
\begin{aligned}
& \frac{1}{2} \times\left[\begin{array}{c}
X_{1 i k} A_{i j}+A_{i j}^{\prime} X_{1 i k}^{\prime}+X_{1 i j} A_{i k}+A_{i k}^{\prime} X_{1 i j}^{\prime} \\
P_{j k}-X_{1 i k}^{\prime}+X_{2 i k} A_{i j}+P_{k j}-X_{1 i j}^{\prime}+X_{2 i j} A_{i k}
\end{array}\right. \\
& \left.\begin{array}{c}
P_{j k}-X_{1 i k}+A_{i j}^{\prime} X_{2 i k}^{\prime}+P_{k j}-X_{1 i j}+A_{i k}^{\prime} X_{2 i j}^{\prime} \\
-X_{2 i k}-X_{2 i k}^{\prime}-X_{2 i j}-X_{2 i j}^{\prime}
\end{array}\right] \\
& <\frac{1}{2} \times\left[\begin{array}{cc}
Q_{0 j k}+Q_{0 k j}+2 C^{\prime} Q_{i} C & 0 \\
0 & 0
\end{array}\right], \quad k \leq j, \\
& Q_{0 j k}+C^{\prime} Q_{\lambda} C<0 \text {, }
\end{aligned}
$$

for all $i \in \mathbb{K}_{N}, j \in \mathbb{K}_{r}$ and $k \in \mathbb{K}_{r}$, then the switching strategy (10) makes the origin $x=0$ of the uncertain switched linear system (6) and (7) a globally asymptotically stable equilibrium point.

Proof Consider that (20) and (21) are feasible. Then, from (10) and (21), for $x \neq 0$, it follows that:

$$
\begin{aligned}
0 & >x^{\prime}\left(Q_{0 j k}+C^{\prime} Q_{\lambda} C\right) x \geq x^{\prime} Q_{0 j k} x+\min _{i \in \mathbb{K}_{N}}\left(y^{\prime} Q_{i} y\right) \\
& =x^{\prime}\left(Q_{0 j k}+C^{\prime} Q_{\sigma} C\right) x .
\end{aligned}
$$

Observe that (22) can be rewritten as:

$$
\begin{aligned}
& x^{\prime}\left(Q_{0 j k}+C^{\prime} Q_{\sigma} C\right) x= \\
& x^{\prime}\left[\begin{array}{ll}
I_{n} & A^{\prime}(\sigma, \alpha)
\end{array}\right]\left[\begin{array}{cc}
Q_{0 j k}+C^{\prime} Q_{\sigma} C & 0 \\
0 & 0
\end{array}\right]\left[\begin{array}{c}
I_{n} \\
A(\sigma, \alpha)
\end{array}\right] x \\
& <0 \text {. }
\end{aligned}
$$

Now, define $P(\alpha)=\left(\alpha_{1} \alpha_{1} P_{11}+\alpha_{1} \alpha_{2} P_{12}+\ldots+\alpha_{r} \alpha_{r} P_{r r}\right)$, $X_{1}(\sigma, \alpha)=\left(\alpha_{1} X_{1 \sigma 1}+\alpha_{2} X_{1 \sigma 2}+\ldots+\alpha_{r} X_{1 \sigma r}\right), X_{2}(\sigma, \alpha)=$ $\left(\alpha_{1} X_{2 \sigma 1}+\alpha_{2} X_{2 \sigma 2}+\ldots+\alpha_{r} X_{2 \sigma r}\right)$. Thus, multiplying (23) by $\alpha_{j} \times \alpha_{k}$ and taking the sum from $j=1$ to $j=r$ and $k=1$ to $k=r$, respectively, from (20), note that:

$$
\begin{aligned}
& 0>\frac{1}{2} \sum_{k=1}^{r} \alpha_{k} \sum_{\substack{j=1 \\
j \geq k}}^{r} \alpha_{j} x^{\prime}\left[\begin{array}{ll}
I_{n} & \left.A^{\prime}(\sigma, \alpha)\right]
\end{array}\right. \\
& {\left[\begin{array}{cc}
Q_{0 j k}+Q_{0 k j}+2 C^{\prime} Q_{\sigma} C & 0 \\
0 & 0
\end{array}\right]\left[\begin{array}{c}
I_{n} \\
A(\sigma, \alpha)
\end{array}\right] x} \\
& >\frac{1}{2} \sum_{k=1}^{r} \alpha_{k} \sum_{\substack{j=1 \\
j \geq k}}^{r} \alpha_{j} x^{\prime}\left[\begin{array}{ll}
I_{n} & \left.A^{\prime}(\sigma, \alpha)\right]
\end{array}\right. \\
& {\left[\begin{array}{c}
X_{1 \sigma k} A_{\sigma j}+A_{\sigma j}^{\prime} X_{1 \sigma k}^{\prime}+X_{1 \sigma j} A_{\sigma k}+A_{\sigma k}^{\prime} X_{1 \sigma j}^{\prime} \\
P_{j k}-X_{1 \sigma k}^{\prime}+X_{2 \sigma k} A_{\sigma j}+P_{k j}-X_{1 \sigma j}^{\prime}+X_{2 \sigma j} A_{\sigma j}
\end{array}\right.} \\
& \left.\begin{array}{c}
P_{j k}-X_{1 \sigma k}+A_{\sigma j}^{\prime} X_{2 \sigma k}^{\prime}+P_{k j}-X_{1 \sigma j}+A_{\sigma k}^{\prime} X_{2 \sigma j}^{\prime} \\
-X_{2 \sigma k}-X_{2 \sigma k}^{\prime}-X_{2 \sigma j}-X_{2 \sigma j}^{\prime}
\end{array}\right] \\
& {\left[\begin{array}{c}
I_{n} \\
A(\sigma, \alpha)
\end{array}\right] x} \\
& =\sum_{k=1}^{r} \alpha_{k} \sum_{j=1}^{r} \alpha_{j} x^{\prime}\left[\begin{array}{ll}
I_{n} & \left.A^{\prime}(\sigma, \alpha)\right]
\end{array}\right. \\
& {\left[\begin{array}{cc}
X_{1 \sigma k} A_{\sigma j}+A_{\sigma j}^{\prime} X_{1 \sigma k}^{\prime} & P_{j k}-X_{1 \sigma k}+A_{\sigma j}^{\prime} X_{2 \sigma k}^{\prime} \\
P_{j k}-X_{1 \sigma k}^{\prime}+X_{2 \sigma k} A_{\sigma j} & -X_{2 \sigma k}-X_{2 \sigma k}^{\prime}
\end{array}\right]} \\
& {\left[\begin{array}{c}
I_{n} \\
A(\sigma, \alpha)
\end{array}\right] x} \\
& =x^{\prime}\left[\begin{array}{ll}
I_{n} & A^{\prime}(\sigma, \alpha)
\end{array}\right] \\
& {\left[X_{1}(\sigma, \alpha) A(\sigma, \alpha)+A^{\prime}(\sigma, \alpha) X_{1}^{\prime}(\sigma, \alpha)\right.} \\
& P(\alpha)-X_{1}^{\prime}(\sigma, \alpha)+X_{2}(\sigma, \alpha) A(\sigma, \alpha) \\
& \left.\begin{array}{c}
P(\alpha)-X_{1}(\sigma, \alpha)+A^{\prime}(\sigma, \alpha) X_{2}^{\prime}(\sigma, \alpha) \\
-X_{2}(\sigma, \alpha)-X_{2}^{\prime}(\sigma, \alpha)
\end{array}\right] \\
& {\left[\begin{array}{c}
I_{n} \\
A(\sigma, \alpha)
\end{array}\right] x} \\
& =x^{\prime}\left[\begin{array}{ll}
I_{n} & A^{\prime}(\sigma, \alpha)
\end{array}\right]\left\{\left[\begin{array}{cc}
0 & P(\alpha) \\
P(\alpha) & 0
\end{array}\right]\right. \\
& +\left[\begin{array}{l}
X_{1}(\sigma, \alpha) \\
X_{2}(\sigma, \alpha)
\end{array}\right]\left[A(\sigma, \alpha)-I_{n}\right] \\
& \left.+\left[\begin{array}{c}
A^{\prime}(\sigma, \alpha) \\
-I_{n}
\end{array}\right]\left[X_{1}^{\prime}(\sigma, \alpha) \quad X_{2}^{\prime}(\sigma, \alpha)\right]\right\}\left[\begin{array}{c}
I_{n} \\
A(\sigma, \alpha)
\end{array}\right] x \\
& =x^{\prime}\left[\begin{array}{ll}
I_{n} & A^{\prime}(\sigma, \alpha)
\end{array}\right]\left[\begin{array}{cc}
0 & P(\alpha) \\
P(\alpha) & 0
\end{array}\right]\left[\begin{array}{c}
I_{n} \\
A(\sigma, \alpha)
\end{array}\right] x \text {. }
\end{aligned}
$$

Considering a Lyapunov function candidate $V(x)=$ $x^{\prime} P(\alpha) x$, note that from (7), $V(x)>0$ for $x \neq 0$ and from (6) and (24), it follows that $\dot{V}(x)<0$ for $x \neq 0$. The proof is concluded. 
The following theorem compares the conditions from Theorems 3 and 5.

Theorem 6 If the conditions given in Theorem 3 hold, then the conditions given in Theorem 5 also hold.

Proof Suppose that the conditions (11) and (12) from Theorem 3 hold for all $i \in \mathbb{K}_{N}$ and $j, k \in \mathbb{K}_{r}$. Then, there exist matrices $Q_{0 j}, Q_{i}, P_{k}$ and $P_{j}$ and a small constant $\xi \in \mathbb{R}$, $\xi>0$, such that

$$
\begin{aligned}
& \frac{1}{2} \times\left\{F_{i j k}-2 Q_{0 j}-2 C^{\prime} Q_{i} C\right. \\
& \left.\quad+\xi\left(A_{i j}^{\prime}+A_{i k}^{\prime}\right)\left(\frac{1}{4} \times \xi^{-1} I_{n}\right)\left(A_{i j}+A_{i k}\right) \xi\right\}<0,
\end{aligned}
$$

where $F_{i j k}=A_{i j}^{\prime} P_{k}+P_{k} A_{i j}+A_{i k}^{\prime} P_{j}+P_{j} A_{i k}$. Now, using the Schur complement (Boyd et al. 1994), then (25) is equivalent to

$$
\frac{1}{2} \times\left[\begin{array}{cc}
Z_{i j k} & \xi\left(A_{i j}^{\prime}+A_{i k}^{\prime}\right) \\
\left(A_{i j}+A_{i k}\right) \xi & -4 \xi I_{n}
\end{array}\right]<0,
$$

where $Z_{i j k}=F_{i j k}-2 Q_{0 j}-2 C^{\prime} Q_{i} C$.

Observe that, for $Q_{0 j k}=Q_{0 k j}=Q_{0 j}, P_{j k}=P_{k}, P_{k j}=$ $P_{j}, X_{1 i k}=P_{k}, X_{1 i j}=P_{j}, X_{2 i j}=\xi I_{n}$ and $X_{2 i k}=\xi I_{n}$, for all $k, j \in \mathbb{K}_{r}$ and $i \in \mathbb{K}_{N}$, if the condition (26) holds, then the condition (20) also holds. The proof is concluded.

Theorem 7 If the conditions given in Theorem 4 hold, then the conditions given in 5 also hold.

Proof Observe that, for $X_{1 i k}=X_{1 i}, X_{2 i k}=X_{2 i}$ and $Q_{0 j k}=Q_{0 j}$, for all $j \in \mathbb{K}_{r}, k \in \mathbb{K}_{r}$ and $i \in \mathbb{K}_{N}$, then the condition (20) is equivalent to the condition (14). Thus, if (14) holds then (20) holds. Finally, note that (15) and (21) are equivalent conditions and the proof is concluded.

Remark 2 The conditions presented in Theorems 2, 3, 4 and 5 are a particular class of BMIs (bilinear matrix inequalities) which contains terms as the product of a scalar by a matrix. Nowadays to the best of the authors' knowledge, there are no solvers that can find solutions for all kinds of BMIs. However, for the class of BMIs obtained in this paper, the path-following method has been used to find feasible solutions (Hassibi et al. 1999; Souza et al. 2014). In Example 1, there is only one bilinear term ( $\lambda_{1}$ because $\left.\lambda_{2}=1-\lambda_{1}\right)$. In this case, the feasible region was obtained for $\lambda_{1} \in\{0.05,0.1,0.15, \ldots, 1\}$. Now in Example 2, there are two bilinear terms $\lambda_{1}$ and $\lambda_{2}$ because $\lambda_{3}=1-\left(\lambda_{1}+\lambda_{2}\right)$. For this case, were adopted $\lambda_{1}=0.1, \lambda_{2}=0.7$ and $\lambda_{3}=0.2$. A future research in this subject is the use of procedures for solving BMIs, as presented in (Hassibi et al. 1999; Souza et al. 2014), for obtaining feasible solutions using the proposed theorems.

\section{Illustrative Examples}

In this section, two examples are used to illustrate the effectiveness of the control method proposed in this paper. More specifically the examples show that the proposed Theorem 5 can ensure feasibility and a suitable performance in situations when the conditions from the proposed Theorems 2, 3 and 4 are not feasible. The simulation results are presented below. The solver in these designs was the LMIlab from the software MATLAB interfaced by the YALMIP (Lofberg 2004).
Fig. 1 Feasible regions obtained with Theorem 2 and Theorem 3, where the region obtained with Theorem 2 is illustrated by filled circle and the region obtained with Theorem 3 is illustrated by filled circle and cross

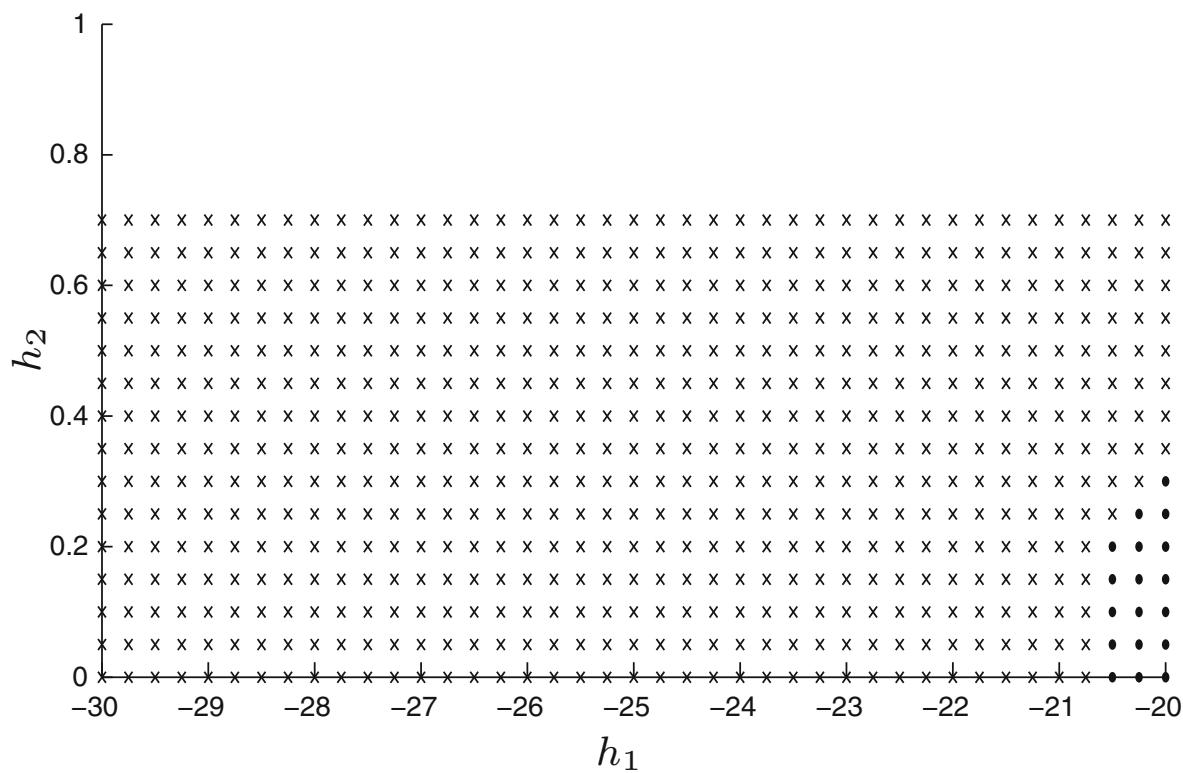


Example 1 Consider the uncertain system (6) and (7), with $r=N=2, i \in\{1,2\}, j \in\{1,2\}$ and the following matrices given below:

$$
\begin{aligned}
& A_{11}=\left[\begin{array}{rrr}
0 & 1 & 0 \\
1 & -3 & 0 \\
1 & 0 & -1
\end{array}\right], A_{21}=\left[\begin{array}{rrr}
-1 & -1.5 & 0 \\
-2 & -1 & 0 \\
0 & 1 & -1
\end{array}\right], \\
& A_{12}=\left[\begin{array}{rrr}
h_{1} & 1 & 0 \\
1 & -1 & 0 \\
1 & 0 & -1
\end{array}\right], A_{22}=\left[\begin{array}{rrr}
-1 & -0.5 & 0 \\
-2 & h_{2} & 0 \\
0 & 1 & -1
\end{array}\right] .
\end{aligned}
$$

Note that the matrices $A_{12}$ and $A_{22}$ depend on the parameters $h_{1}$ and $h_{2}$. It is interesting to observe that the matrix $A_{12}$ is not Hurwitz for all $h_{1} \in[-30,-20]$ and the matrix $A_{22}$ is Hurwitz for all $h_{2} \in[0,1]$. Furthermore, note that the matrices $A_{11}$ and $A_{21}$ are not Hurwitz. This example presents a comparative study of the feasibility, regarding the conditions of the proposed theorems, for some pairs $\left(h_{1}, h_{2}\right)$, where $h_{1} \in[-30,-20]$ and $h_{2} \in[0,1]$. The output matrix of the uncertain switched linear system is defined by:

$C=\left[\begin{array}{lll}1 & 0 & 0 \\ 0 & 0 & 1\end{array}\right]$.

In this example, first we adopted $\lambda_{1}=\lambda_{2}=0.5$. Considering only feasibility, Fig. 1 illustrates a comparison between the conditions from Theorems 2 and 3.

Note that, in Fig. 1, the feasible region obtained with Theorem 3 is greater than the feasible region obtained with Theorem 2.

Remark 3 It is important to highlight that in this example, the values of $\lambda_{1}$ and $\lambda_{2}$ were determined based on the feasibility
Fig. 2 Feasible regions obtained with Theorems 3 and 4 , where the region obtained with Theorem 3 is illustrated by cross and the region obtained with Theorem 4 is illustrated by cross and open circle

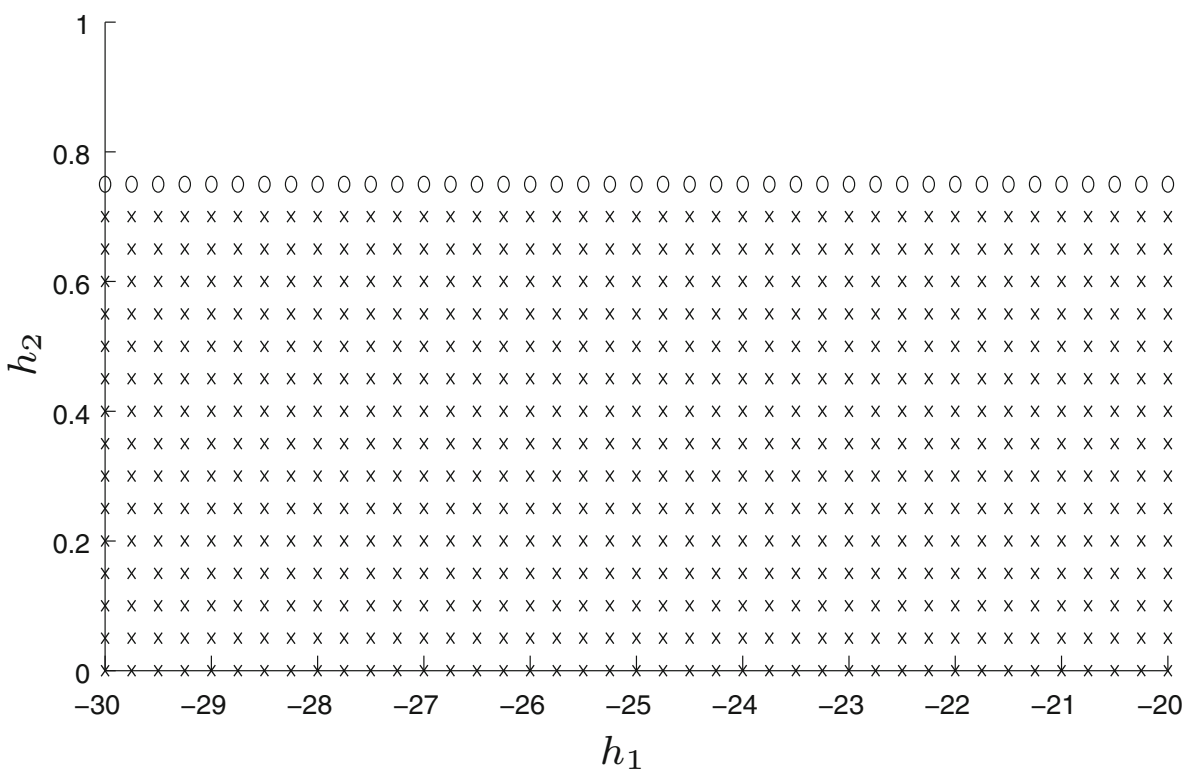

Fig. 3 Feasible regions obtained with Theorems 4 and 5, where the region obtained with Theorem 4 is illustrated by open circle and the region obtained with Theorem 5 is illustrated by open circle and filled circle

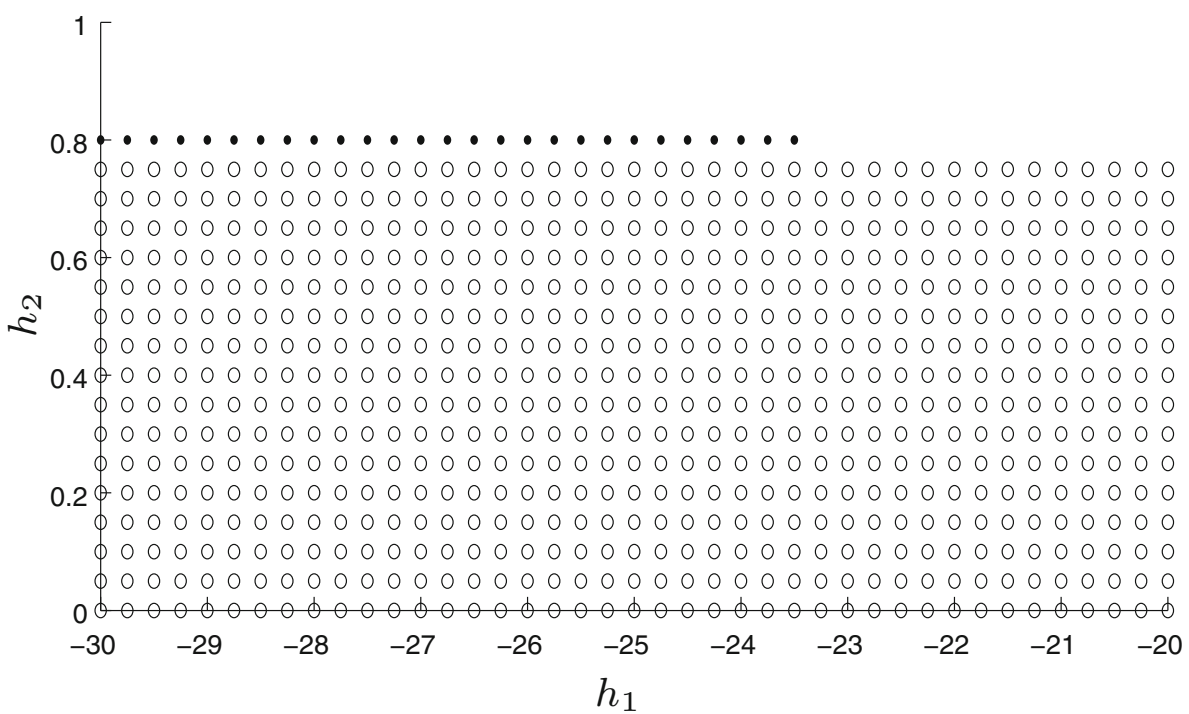


Fig. 4 Feasible regions obtained with Theorem 4, where the region obtained with $\lambda_{1}=0.35$ is illustrated by filled circle, with $\lambda_{1}=0.5$ is illustrated by filled circle and cross and with $\lambda_{1}=0.65$ is illustrated by filled circle, cross and open circle

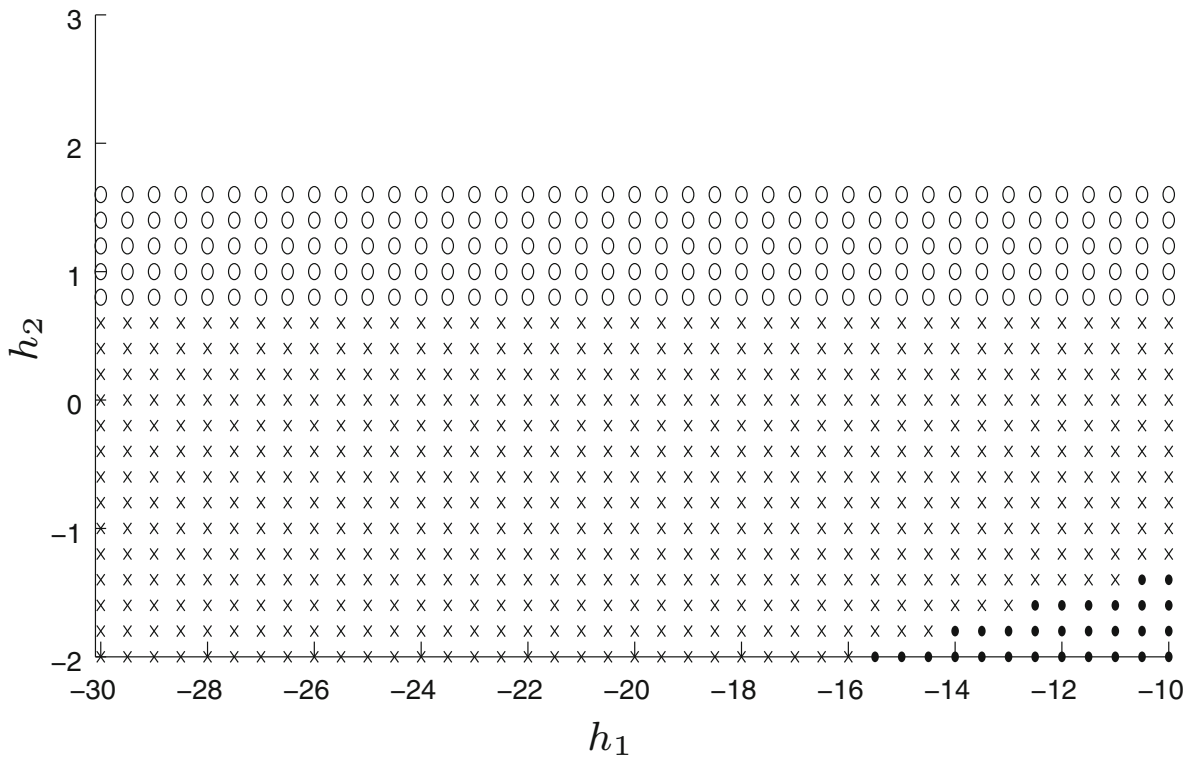

Fig. 5 Feasible regions obtained with Theorem 5, where the region obtained with $\lambda_{1}=0.35$ is illustrated by filled circle, with $\lambda_{1}=0.5$ is illustrated by filled circle and cross and with $\lambda_{1}=0.75$ is illustrated by filled circle, cross and open circle

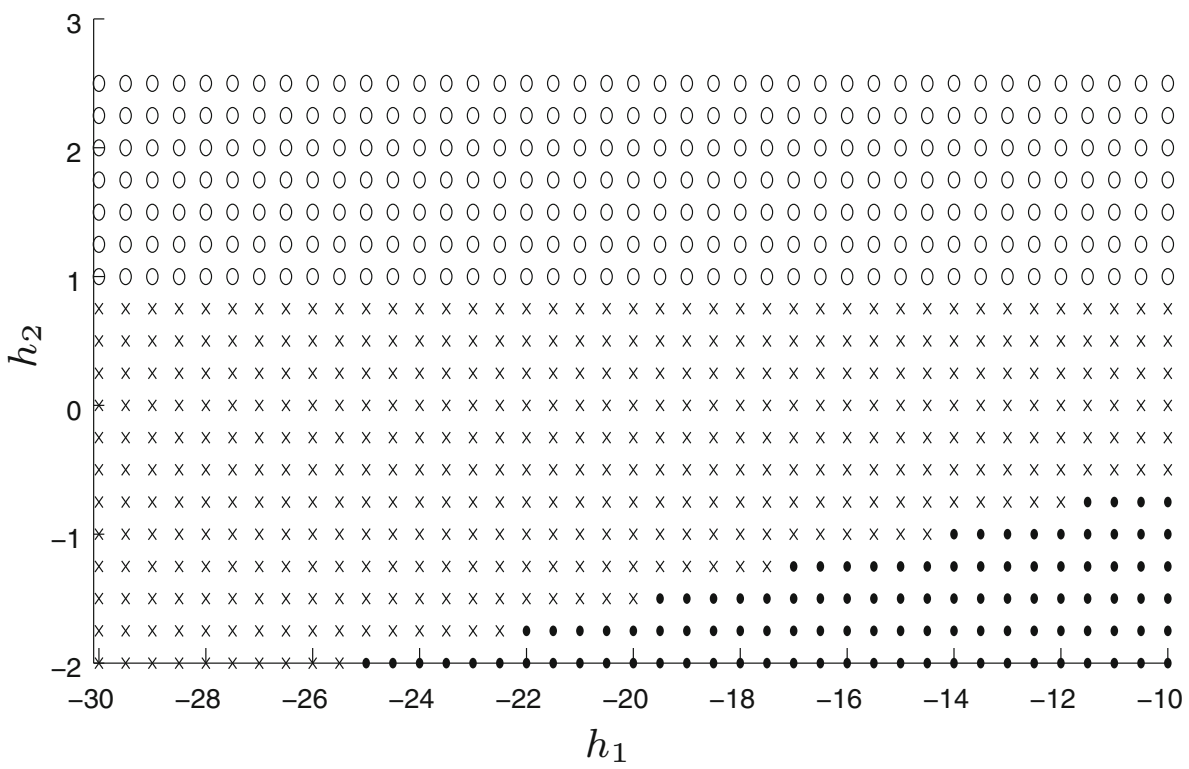

region obtained by Theorem 2 . More specifically, considering that $\lambda_{1}$ belongs to the set $[0,0.05,0.1, \ldots, 0.95,1]$, one obtains feasibility with the conditions from Theorem 2 , only for $\lambda_{1}=\lambda_{2}=0.5$.

Now, for the same parameters defined above, consider $h_{1}$ and $h_{2}$ belonging to the sets $h_{1} \in[-30,-20]$ and $h_{2} \in$ $[0,1]$. Then, Fig. 2 illustrates a comparison between the conditions from Theorems 3 and 4.

From Fig. 2, observe that the set of slack variables introduced in Theorem 4 reduces the conservatism and expands the stability margin.

Figure 3 shows the comparison between Theorems 4 and 5 . Note that the proposed condition given by Theorem 5 involves all previous ones with a larger stability region. This fact illustrates the results presented in Theorem 7 .
In order to find the optimal solution based on the parameters of the convex combination, $\lambda \in \Lambda$, the feasibility region of Theorems 4 and 5 is computed considering $\lambda_{i} \geq 0$, $i \in \mathbb{K}_{N}$, such that $\lambda_{1}+\lambda_{2}+\ldots+\lambda_{N}=1$. Then, consider $h_{1}$ and $h_{2}$ belonging to the sets $h_{1} \in[-30,-10]$ and $h_{2} \in[-2,3]$. In Figs. 4 and 5, are presented three feasible areas: the $\lambda_{1}$ that holds the lesser region of feasibility, the $\lambda_{1}$ that ensures the greatest region of feasibility and the $\lambda_{1}$ previously adopted in this example $\left(\lambda_{1}=\lambda_{2}=\right.$ $0.5)$.

From Fig. 4, note that for $\lambda_{1}=0.65$ and $\lambda_{2}=0.35$, Theorem 4 presents the greater feasibility region.

As shown in Fig. 5, observe that the greater feasibility region for Theorem 5 is obtained with $\lambda_{1}=0.75$ and $\lambda_{2}=$ 0.25 . Moreover, in both cases, the simulation results showed 
Fig. 6 Feasible regions obtained with Theorems 2 and 3, where the region obtained with Theorem 2 is illustrated by filled circle and the region obtained with Theorem 3 is illustrated by filled circle and cross

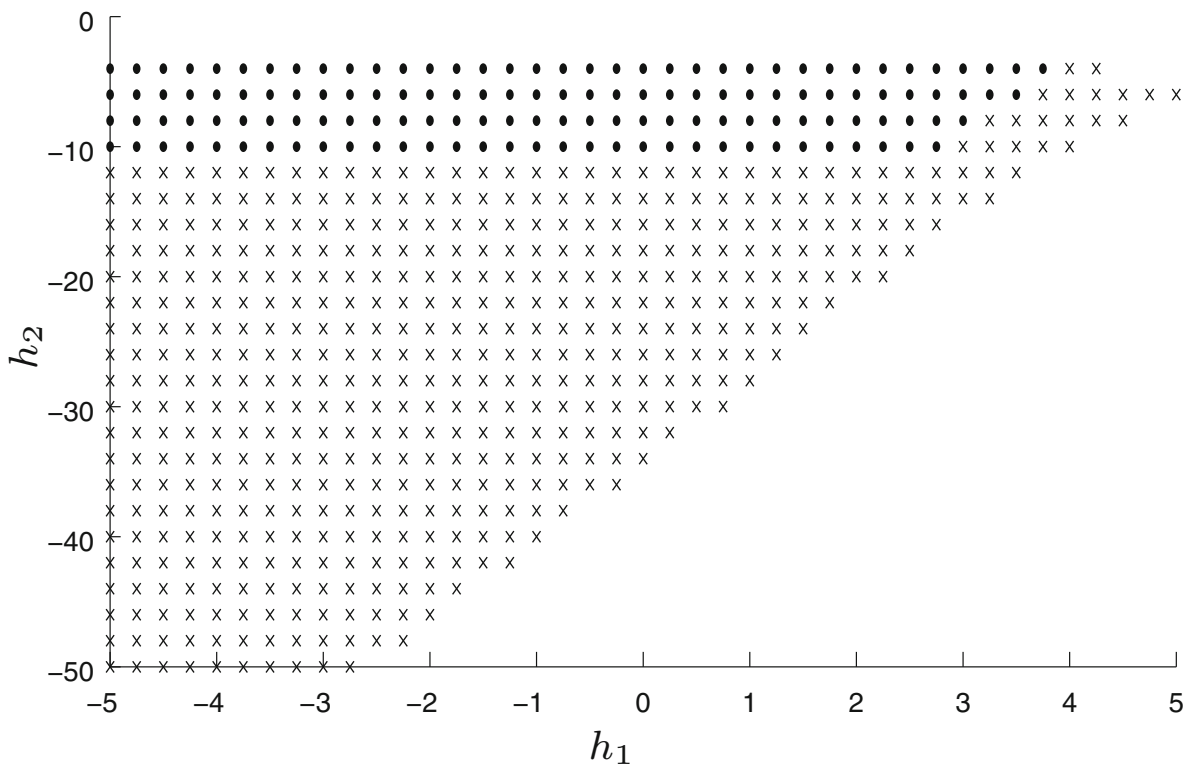

Fig. 7 Feasible regions obtained with Theorems 3 and 4 , where the region obtained with Theorem 3 is illustrated by crossand the region obtained with Theorem 4 is illustrated by crossand open circle

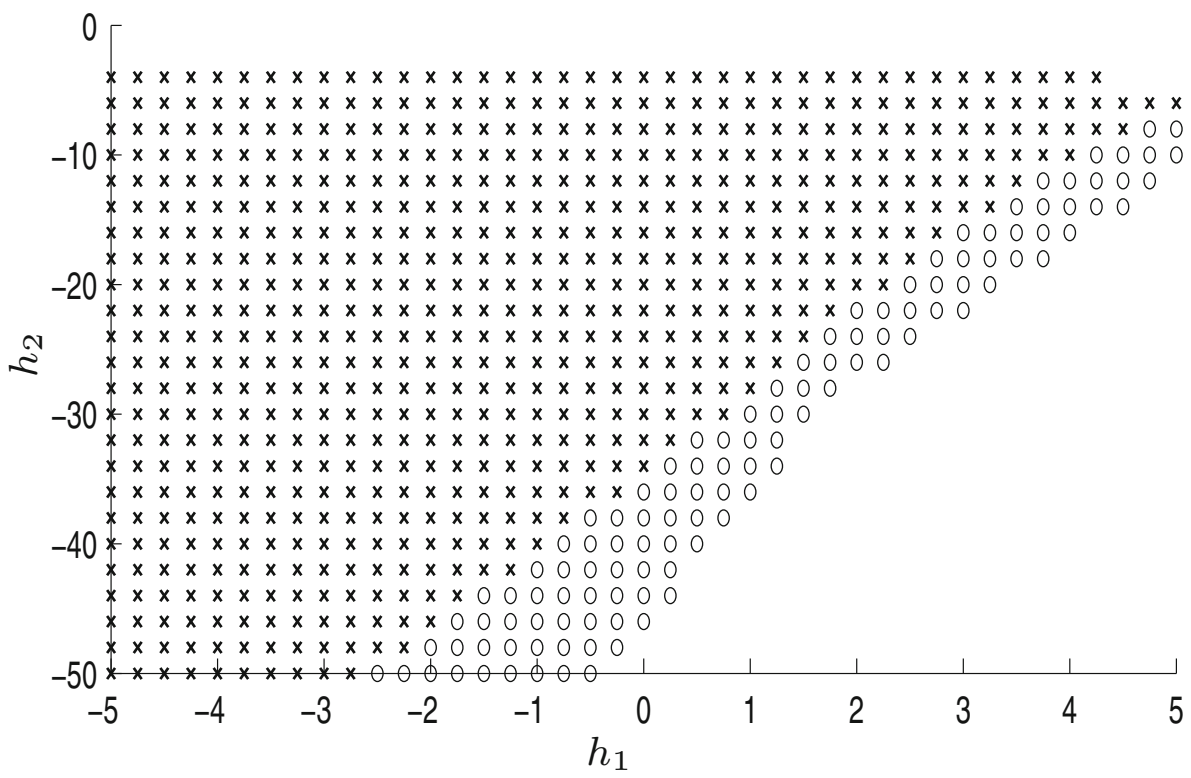

that the minimum value of $\lambda_{1}$ that ensures feasibility was $\lambda_{1}=0.35\left(\lambda_{2}=0.65\right)$.

Example 2 In this example, consider the uncertain system (6) and (7), with $r=2, N=3, i \in\{1,2,3\}, j \in\{1,2\}$ and the following matrices given below:

$$
\begin{aligned}
& A_{11}=\left[\begin{array}{rrr}
2 & 1 & 0 \\
3 & -5 & 0 \\
2 & 0 & -2
\end{array}\right], A_{12}=\left[\begin{array}{rrr}
h_{1} & 1 & 0 \\
3 & -2 & 0 \\
2 & 0 & -2
\end{array}\right] \\
& A_{21}=\left[\begin{array}{rrr}
-5 & -3 & 1 \\
-3 & -2 & 0 \\
0 & 2 & -2
\end{array}\right], A_{22}=\left[\begin{array}{rrr}
-5 & -6 & 1 \\
-3 & h_{2} & 0 \\
0 & 2 & -2
\end{array}\right],
\end{aligned}
$$

$$
A_{31}=\left[\begin{array}{rrr}
0 & 1 & 0 \\
1 & -2 & 0 \\
3 & 0 & -3
\end{array}\right], A_{32}=\left[\begin{array}{rrr}
0 & 1 & -3 \\
1 & -2 & 0 \\
3 & h_{3} & -3
\end{array}\right]
$$

Note that the matrices $A_{12}, A_{22}$ and $A_{32}$ depend on the parameters $h_{1}, h_{2}$ and $h_{3}$, respectively. It is interesting to observe that the matrices $A_{12}$ and $A_{32}$ are not Hurwitz for all $h_{1} \in[-5,-5]$ and $h_{3} \in[-2.5,27.5]$, respectively, and the matrix $A_{22}$ is Hurwitz for all $h_{2} \in[-50,0]$. Furthermore, note that the matrices $A_{11}$ and $A_{31}$ are not Hurwitz and the matrix $A_{21}$ is Hurwitz.

This example presents a comparative study of the feasibility, regarding the conditions of the proposed theorems, 
Fig. 8 Feasible regions obtained with Theorems 4 and 5, where the region obtained with Theorem 4 is illustrated by open circle and the region obtained with Theorem 5 is illustrated by open circle and filled circle

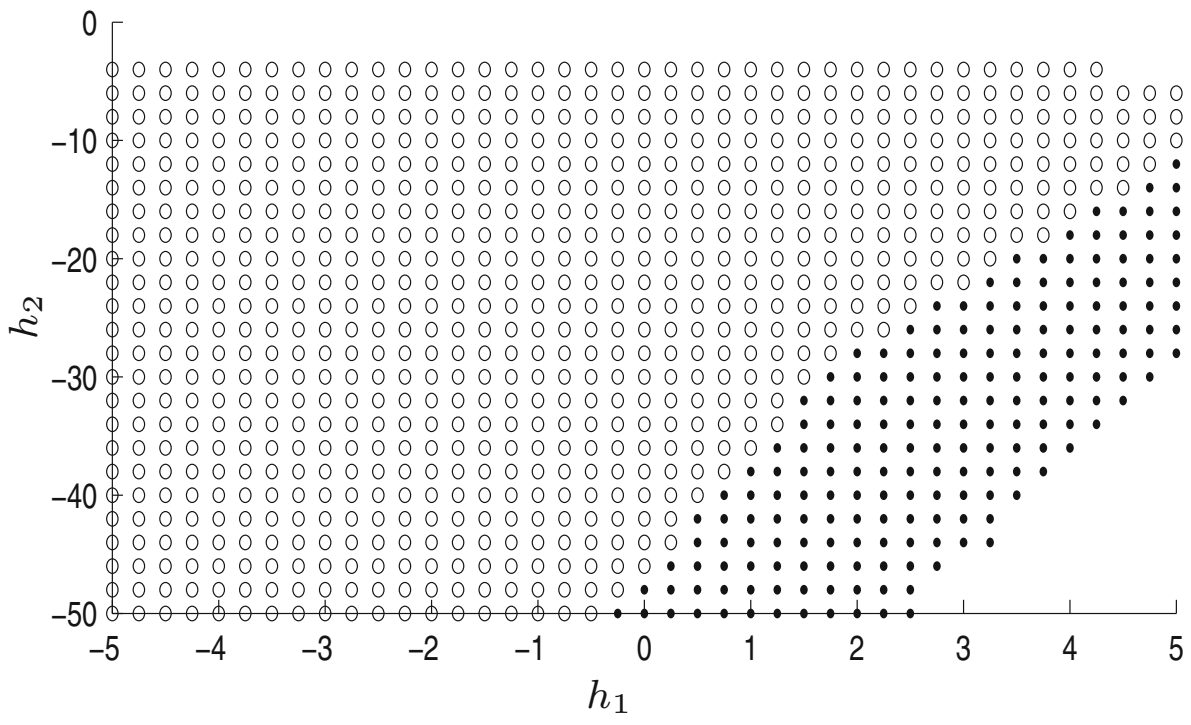

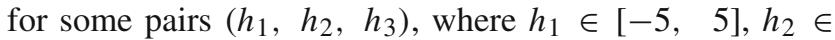
$[-50,0]$ and $h_{3}=-\left(h_{1}+h_{2}\right) / 2$. The output matrix of the uncertain switched linear system is:

$C=\left[\begin{array}{lll}0 & 1 & 0 \\ 0 & 0 & 1\end{array}\right]$.

Initially, in this example were adopted $\lambda_{1}=0.1, \lambda_{2}=0.7$ and $\lambda_{3}=0.2$. Figure 6 illustrates a comparison between the conditions from Theorems 2 and 3 . Note that in the first example, the feasible region obtained with Theorem 3 is greater than the feasible region obtained with Theorem 2 .

Now, for the same parameters defined above, Fig. 7 illustrates a comparison between the conditions from Theorems 3 and 4 . Then, observe that the proposed condition given by Theorem 4 involves all previous ones with a larger stability region.

Figure 8 shows the comparison between Theorems 4 and 5. From Fig. 8, note that Theorem 5 presents the greater feasibility region than Theorem 4 .

\section{Conclusion}

This paper proposed a control design method for continuoustime uncertain switched linear systems using only a switching strategy that depends on the plant output. The design was based on quadratic Lyapunov functions and LMIs. New less conservative conditions were obtained. From Theorems 6 and 7, if the conditions given in Theorems 2, 3 and 4 hold, then the conditions given in Theorem 5 also hold. This fact was illustrated in the simulations results presented in examples. However, the simulations showed that when feasible, the proposed Theorem 5 presented a greater feasibility region than other theorems. Additionally, from Example 1, through search results of the optimal $\lambda \in \Lambda$, the greatest feasibility area with the conditions from Theorem 5 was obtained for $\lambda_{1}=0.65$ and $\lambda_{2}=0.35$, showing the effectiveness of proposal control method. A future research in this subject is the use of the path-following method in the design procedure, as detailed in Remark 2. Moreover, aiming to improve the feasibility of the proposed Theorems, the design can be based on minimum-type Lyapunov functions as presented in Geromel and Colaneri (2006).

Acknowledgments The authors gratefully acknowledge the financial support by CAPES, CNPq and FAPESP from Brazil (Process: 2012/12945-7 linked to the Thematic Project—Process: 2011/17610-0) for supporting this work.

\section{References}

Bernussou, J., Peres, P. L. D., \& Geromel, J. C. (1989). A linear programming oriented procedure for quadratic stabilization of uncertain systems. Systems and Control Letters, 13(1), 65-72.

Boyd, S., Ghaoui, L., Feron, E., \& Balakrishnan, V. (1994). Linear matrix inequalities in systems and control theory. Studies in Applied Mathematics (Vol. 15). Philadelphia, PA: SIAM

Cardim, R., Teixeira, M. C. M., Assunção, E., \& Covacic, M. R. (2009). Variable-structure control design of switched systems with an application to a DC-DC power converter. IEEE Transaction on Industrial Electronics, 56(9), 3505-3513.

da Cruz Figueredo, L. F., Ishihara, J. Y., Borges, G. A., \& Bauchspiess, A. (2013). Delay-dependent robust stability analysis for time-delay T-S fuzzy systems with nonlinear local models. Journal of Control, Automation and Electrical Systems, 24(1-2), 11-21.

Daafouz, J., Riedinger, P., Claude, I. (2001). Static output feedback control for switched systems. In: Proceedings of the 40th IEEE conference on decision and control, 3, 2093-2094. 
Daafouz, J., Riedinger, P., \& Iung, C. (2002). Stability analysis and control synthesis for switched systems: A switched Lyapunov function approach. IEEE Transactions on Automatic Control, 47(11), 1883-1887.

Deaecto, G. S., Geromel, J. C., Garcia, F. S., \& Pomilio, J. A. (2010). Switched affine systems control design with application to DCDC converters. IET Control Theory and Applications, 4(7), 12011210.

Deaecto, G. S., Geromel, J. C., \& Daafouz, J. (2011). Dynamic output feedback $\mathcal{H}_{\infty}$ control of switched linear systems. Automatica, 47(8), 1713-1720.

Decarlo, R. A., Branicky, M. S., Pettersson, S., \& Lennartson, B. (2000). Perspectives and results on the stability and stabilizability of hybrid systems. Proceedings of the IEEE, 88(7), 1069-1082.

Ding, D., \& Yang, G. H. (2009). Static output feedback control for discrete-time piecewise linear systems: An LMI approach. Acta Automatica Sinica, 35(4), 337-344.

Dong, J., \& Yang, G. H. (2007). Static output feedback control synthesis for linear systems with time-invariant parametric uncertainties. IEEE Transactions on Automatic Control, 52(10), 1930-1936.

Feron, E. (1996). Quadratic stabilizability of switched systems via state and output feedback. Cambridge: Center for Intelligent Control Systems, MIT Publication CICS-P. 1996.

Gahinet, P., Nemirovski, A., Laub, A.J., Chilali, M. (1995). LMI control toolbox-for use with MATLAB, 1995.

Geromel, J. C., \& Colaneri, P. (2006). Stability and stabilization of continuous-time switched linear systems. SIAM, Journal Control Optimization, 45, 1915-1930.

Geromel, J. C., de Souza, C. C., \& Skelton, R. E. (1998). Static output feedback controllers: Stability and convexity. IEEE Transactions on Automatic Control, 43(1), 120-125.

Geromel, J. C., Colaneri, P., \& Bolzern, P. (2008). Dynamic output feedback control of switched linear systems. IEEE Transactions on Automatic Control, 53(3), 720-733.

Guedes, J. A., Teixeira, M. C. M., Cardim, R., \& Assunção, E. (2013). Stability of nonlinear system using Takagi-Sugeno fuzzy models and hyper-rectangle of LMIs. Journal of Control, Automation and Electrical Systems, 24(1-2), 46-53.

Hassibi, A., How, J., Boyd, S. (1999). A path-following method for solving BMI problems in control. In: Proceedings of the 1999, American control conference, 1999., 2, 1385-1389.

Hespanha, J. P., \& Morse, A. S. (2002). Switching between stabilizing controllers. Automatica, 38, 1905-1917.

Iwasaki, T., Skelton, R. E., \& Geromel, J. C. (1994). Linear quadratic suboptimal control with static output feedback. Systems and Control Letters, 23(6), 421-430.

Ji, Z., Long, W., Guangming, X. (2003). New results on the quadratic stabilization of switched linear systems. In: Proceedings 42nd IEEE conference on decision and control, 2, (pp. 1657-1662), 2003.

Liberzon, D. (2003). Switching in systems and control. Birkhuser: Systems \& Control.
Lin, H., \& Antsaklis, P. J. (2007). Switching stabilizability for continuous-time uncertain switched linear systems. IEEE Transactions on Automatic Control, 52(4), 633-646.

Lin, H., \& Antsaklis, P. J. (2009). Stability and stabilizability of switched linear systems: A survey of recent results. IEEE Transactions on Automatic Control, 54(2), 308-322.

Lofberg, J. (2004). YALMIP: A toolbox for modeling and optimization in MATLAB. In: IEEE international symposium on computer aided control systems design, (pp. 284-289), 2004.

Mainardi Júnior, EI., Teixeira, M. C. M., Moreira, M. R., Cardim, R., Assunção, E., Yoshimura, V. L. (2012b). Controle via realimentação da saída para sistemas lineares chaveados. Congresso Brasileiro de Automática, 2012.

Mainardi Júnior, EI., Teixeira, M.C.M., Moreira, M.R., Cardim, R., Assunção, E., de Oliveira, D.R. (2014). Robust control of switched linear systems with output switching strategy. Congresso Brasileiro de Automática, 2014.

Mainardi Júnior EI, Teixeira, M.C.M., Moreira, M.R., Cardim, R., Assunção, E., Yoshimura, V.L. (2012a). On control design of switched affine systems with application to DC-DC converters. Frontiers in Advanced Control Systems, In-Teh, 2012.

Otsuka, N., \& Soga, T. (2010). Quadratic stabilizability for polytopic uncertain continuous-time switched linear systems composed of two subsystems. International Journal of Control and Automation, $3(1), 35-42$.

Scharlau, C. C., de Oliveira, M. C., Trofino, A., \& Dezuo, T. J. M. (2014). Switching rule design for affine switched systems using a max-type composition rule. Systems and Control Letters, 68, 1-8.

Souza, W., Teixeira, M., Cardim, R., \& Assunção, E. (2014). On switched regulator design of uncertain nonlinear systems using Takagi-Sugeno fuzzy models. IEEE Transactions on Fuzzy Systems, 22(6), 1720-1727.

Sun, Z., \& Ge, S. S. (2005a). Analysis and synthesis of switched linear control systems. Automatica, 41(2), 181-195.

Sun, Z., \& Ge, S. S. (2005b). Switched linear systems: Control and design. London: Springer.

Syrmos, V., Abdallah, C., Dorato, P. (1994). Static output feedback: A survey. In: Proceedings of the 33rd IEEE conference on decision and control, 1, (pp. 837-842).

Wicks, M.A., Peleties, P., DeCarlo, R.A. (1994). Construction of piecewise Lyapunov functions for stabilizing switched systems. In: Proceedings of the 33rd IEEE conference on decision and control, 4, (pp. 3492-3497), 1994.

Yoshimura, V. L., Assunção, E., da Silva, E. R., Teixeira, M. C. M., \& Mainardi Júnior, E. I. (2013). Observer-based control design for switched affine systems and applications to DC-DC converters. Journal of Control, Automation and Electrical Systems, 24(4), 535-543.

Zhai, G., Lin, H., \& Antsaklis, P. (2003). Quadratic stabilizability of switched linear systems with polytopic uncertainties. International Journal of Control, 76(7), 747-753. 